\title{
Impact of patient-specific morphologies on sinus flow stasis in transcatheter aortic valve replacement: An in vitro study
}

\author{
Hoda Hatoum, MS, ${ }^{\mathrm{a}}$ Jennifer Dollery, RN, ${ }^{\mathrm{b}}$ Scott M. Lilly, MD, PhD, ${ }^{\mathrm{c}}$ Juan Crestanello, $\mathrm{MD},{ }^{\mathrm{b}}$ and \\ Lakshmi Prasad Dasi, PhD ${ }^{\mathrm{a}, \mathrm{b}}$
}

\begin{abstract}
Objective: The goal of this study is to evaluate how sinus flow patterns after transcatheter aortic valve replacement in realistic representative patient roots vary. Sinus flow can affect transcatheter aortic valve operation and likely leaflet thrombosis occurrence due to stasis and poor washout. How the interaction between transcatheter aortic valve and representative patient aortic roots affects sinus hemodynamics is important to establish for future individualization of transcatheter aortic valve replacement therapy.
\end{abstract}

Methods: Two representative patient aortic roots were selected, segmented and 3-dimensional printed followed by deployment of Medtronic CoreValve (Medtronic Inc, Irvine, Calif) and Edwards SAPIEN (Edwards Lifesciences, Irvine Calif) transcatheter aortic valves. Sinus hemodynamics were assessed in vitro using high spatio-temporal resolution particle-image-velocimetry. Detailed sinus vortex tracking, shear stress probability density functions, and sinus washout were evaluated and assessed as a function of valve type and representative patient morphology as independent case studies.

Results: Peak velocity in the sinus with SAPIEN valve was approximately 3 times higher than with CoreValve for both models $(0.30 \pm 0.02 \mathrm{~m} / \mathrm{s}$ and $0.34 \pm 0.041 \mathrm{~m} / \mathrm{s}$ vs $0.13 \pm 0.01 \mathrm{~m} / \mathrm{s}$ and $0.10 \pm 0.02 \mathrm{~m} / \mathrm{s})(P<.01)$. Between representative patient models, vorticity magnitudes were significantly different $\left(75 \pm 1.1 \mathrm{~s}^{-1}, 77 \pm 3.2 \mathrm{~s}^{-1}, 109 \pm 2.3 \mathrm{~s}^{-1}\right.$, and $\left.250 \pm 4.1 \mathrm{~s}^{-1}\right)(P<.01)$ regardless of valve type. Sinus blood washout characteristic as a function of cardiac cycles was strongly both patient related and valve specific. Fluid dynamics favored shear stresses and washout characteristics due to a smaller sinus and sinotubular junction, further amplified by the SAPIEN valve.

Conclusions: Sinus flow dynamics are highly sensitive to aortic root characteristics and transcatheter aortic valve aortic root interaction. Differences in sinus-flow washout and stasis regions between representative patient models may be reflected in different risks of leaflet thrombosis or valve degeneration. (J Thorac Cardiovasc Surg 2019;157:540-9)

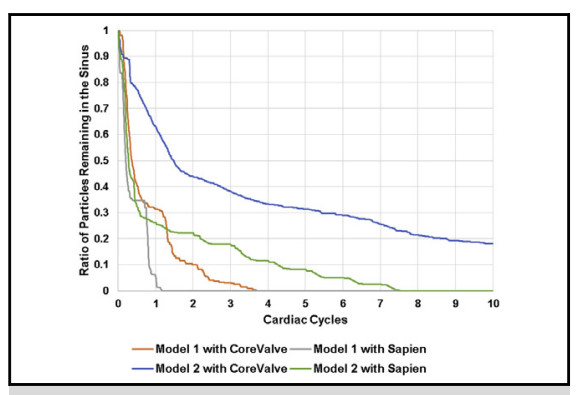

Survival curves of blood particles remaining in sinuses post-TAVR with different valves.

\section{Central Message}

Sinus flow dynamics are highly sensitive to aortic root patient-specificity and TAV aortic root interaction. This may be reflected in different leaflet thrombosis risks.

\section{Perspective}

The aim of this study is to present a detailed picture of sinus blood flow patterns and hemodynamics post-TAVR using realistic patient-specific aortic roots and illustrate how statistical measures that may be predictive of thrombus formation are sensitive not only to the TAV type but also the patient's aortic root morphology itself.

See Editorial Commentary page 550.
From the ${ }^{\mathrm{a}}$ Department of Biomedical Engineering, ${ }^{\mathrm{b}}$ Division of Cardiac Surgery, Department of Surgery, and ${ }^{\mathrm{c}}$ Division of Cardiovascular Medicine, Department of Internal Medicine, The Ohio State University, Columbus, Ohio.

Funding: The research done was partly supported by the National Institutes of Health under Award Number R01HL119824.

Received for publication Jan 17, 2018; revisions received May 8, 2018; accepted for publication May 9, 2018; available ahead of print July 3, 2018.

Address for reprints: Lakshmi Prasad Dasi, PhD, Department of Biomedical Engineering, The Ohio State University, 473 W 12th Ave, Columbus, OH 43210 (E-mail: lakshmi.dasi@osumc.edu).

0022-5223/\$36.00

Copyright (c) 2018 by The American Association for Thoracic Surgery

https://doi.org/10.1016/j.jtcvs.2018.05.086
The primary hemodynamic feature of the aortic sinus that influences aortic valve dynamics, progression of disease, or thrombosis through sinus washout ${ }^{1,2}$ is the aortic sinus vortex. Sinus flow characteristics after transcatheter aortic

- Scanning this $\mathrm{QR}$ code will take you to a supplemental video for the article. 

Abbreviations and Acronyms
LVOT $=$ left ventricular outflow tract
PIV = particle image velocimetry
STJ = sinotubular junction
$\mathrm{TAV}=$ transcatheter aortic valve
TAVR $=$ transcatheter aortic valve replacement

valve replacement (TAVR) procedures can affect the degree of flow stasis around the prosthesis. ${ }^{3-6}$ In our previous study, ${ }^{3}$ we demonstrated that sinus flow patterns were greatly altered once a transcatheter aortic valve (TAV), whether CoreValve (Medtronic Inc, Irvine, Calif) or SAPIEN (Edwards Lifesciences, Irvine Calif), was introduced as valve-in-valve implantation compared with a bioprosthetic surgical valve. ${ }^{3}$ Specifically, peak sinus flow velocity was decreased and a reduced sinus flow manifested toward the sinus base in the valve-in-valve (ViV) cases. ${ }^{3}$ These flow patterns were attributed to the interaction of stent posts with sinus flow, the relative location of leaflet tip with respect to the sinotubular junction (STJ), and other characteristics of the TAV prosthesis. ${ }^{3,5}$ Nevertheless, in routine TAVR, the mechanisms are more complex because of the interaction of TAVs with the representative patient geometry of the aortic root with little current knowledge existing about sinus flow hemodynamics, specifically with respect to flow stasis and washout after routine TAVR.

Flow stasis, expressed by low shear stress or prolonged blood flow residence time, has long been correlated with thrombus formation, and several attempts to define shear stress thresholds for the onset of thrombus formation were and are still being done. ${ }^{7-9}$ In recent publications by Makkar and colleagues ${ }^{10}$ and Chakravarty and colleagues, ${ }^{11}$ reduced leaflet mobility due to thrombus formation on the leaflets post-TAVR was identified. Although it is clear that flow stasis and poor washout are causes for leaflet thrombosis, it is not clear whether these occurrences can be attributed to the valve alone or the interaction of the valve with the surrounding native leaflets or the complex interaction between the valve and the root that dictates the aortic sinus flow. How much of the sinus flow hemodynamics is dictated by the morphology of the sinus itself compared with the type of valve?

Sinus flow dynamics were studied by several research groups in terms of TAV deployment positions, valve types, valve sizes, and valve-in-valve configurations. $2,4,12-14$ Portions of the sinus were also highlighted, such as the neo-sinus, ${ }^{4}$ and the full sinus domain was covered only in idealized nonanatomic and mostly axisymmetric models. ${ }^{12,14}$ Unfortunately, the anatomic morphology of the sinus and aortic root was not examined in pre-TAVR or post-TAVR, and studying it is crucial to determine how sensitive sinus flow hemodynamics is on the root morphology itself.

The aim of the present study is to present a detailed picture of sinus blood flow patterns and hemodynamics post-TAVR using realistic representative patient aortic roots and illustrate how statistical measures that may be predictive of thrombus formation are sensitive not only to the TAV type but also the patient's aortic root morphology itself. This is achieved using state-of-the-art high spatio-temporal resolution hemodynamic measurements toward better furthering our understanding of the mechanisms of sinus flow stasis post-TAVR and inform future individualization of TAVR therapy. Video 1 summarizes the point of the article.

\section{MATERIALS AND METHODS}

\section{Two Distinct Aortic Root Geometries Modeling}

Patients with severe degenerative aortic stenosis being evaluated for TAV replacement at the Ohio State University Wexner Medical Center were selected as part of an Institutional Review Board-approved study. The high spatial resolution of the computed tomography imaging data provide clear depiction of the aortic valve cusps and calcific regions. Two patients with a clinical diagnosis of severe aortic stenosis (bicuspid aortic valve (model 1) with fusion of right and left coronary sinus leaflets and 1 patient with a tricuspid aortic valve (model 2) were selected for anatomic modeling to represent 2 samples of different and wide ranges of aortic sinus root geometries. Each patient provided informed written consent to participate in the study as approved by the Institutional Review Board.

Contrast-enhanced computed tomography DICOM images at the $70 \%$ or $85 \%$ phase were imported into anatomic modeling software (Mimics, Materialise, Belgium) for each patient (Figure 1, A). The left ventricular outflow tract (LVOT), valve cusps, ascending aorta, and all calcified tissues were segmented individually and then reconstructed into a model consisting of 2 paired stereolithographic files composed of the calcified and noncalcified structures within the data set. These stereolithographic files were exported to a Stratasys Connex Printer where the 2 files were used to create a fused material 3-dimensional construct (Figure 1, B and $C$ ) of the predefined anatomic region. Cusp calcification was replicated using rigid print material (VeroWhite clear; Stratasys, Farmington Hills, Mich) and soft tissue structures, including the noncalcified cusp segments, LVOT, and ascending aorta, were replicated using a rubber-like material (TangoPlus FLX930, Stratasys). Each model was coated externally with a thin layer of silicone to improve visual clarity and durability (Figure 1, $D$ and $E$ ). Print material properties were chosen to best represent

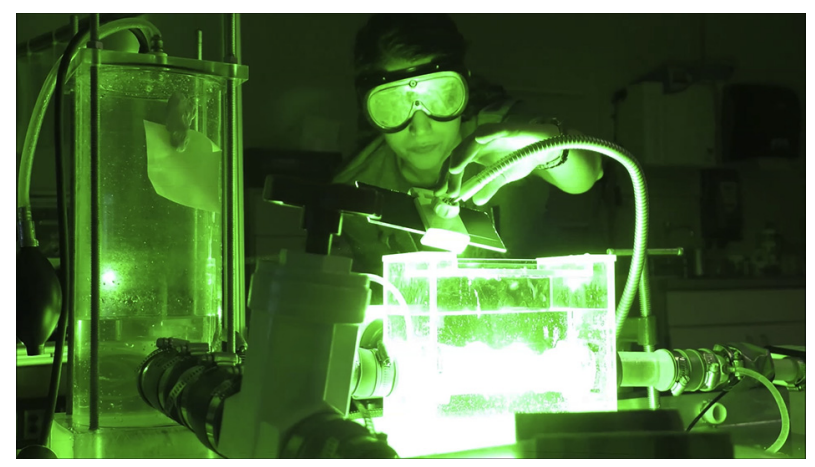

VIDEO 1. Short video summary of the article with narration. Video available at: https://www.jtcvs.org/article/S0022-5223(18)31521-6/fulltext. 

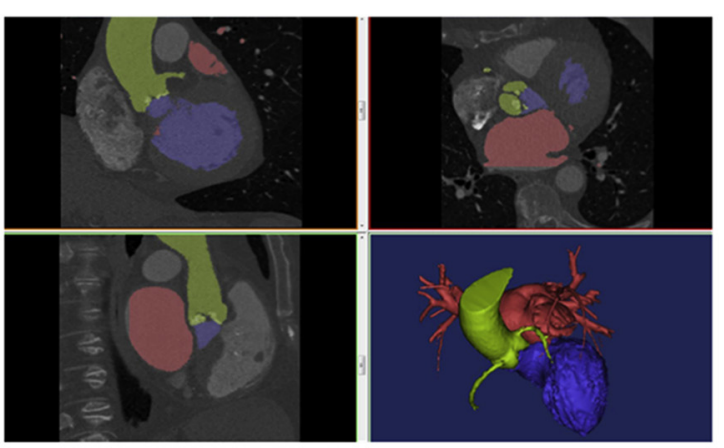

A

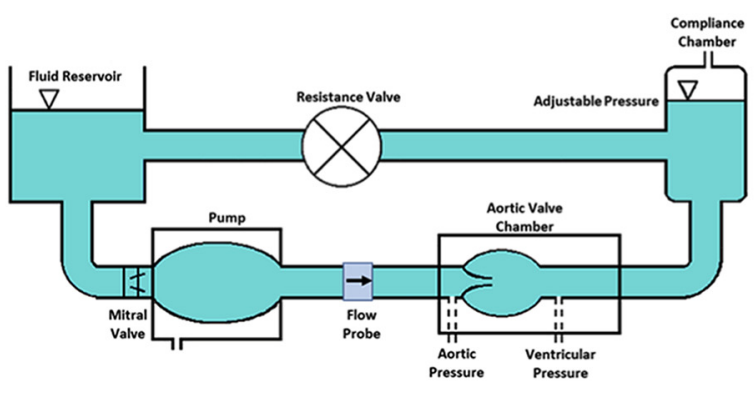

$\mathbf{F}$

E

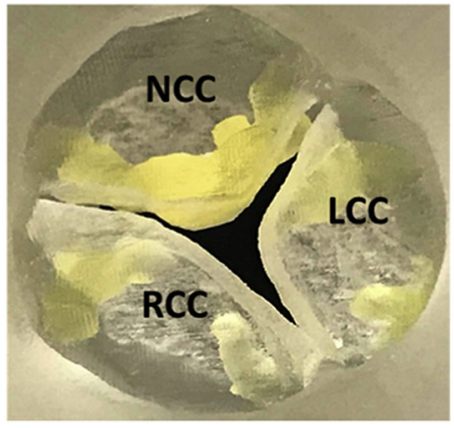

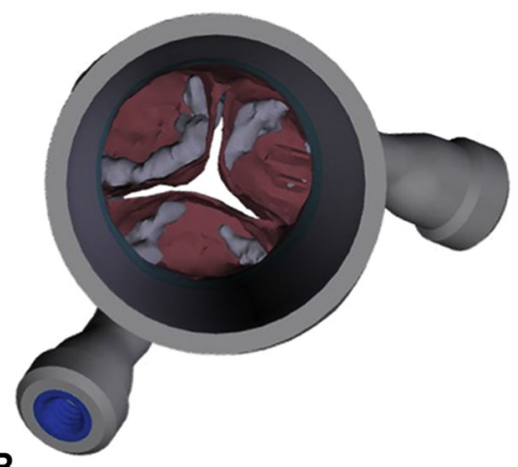

B

C

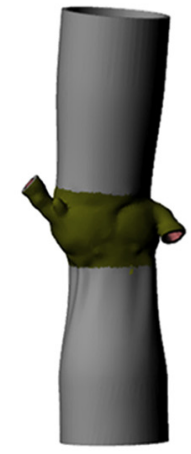

C

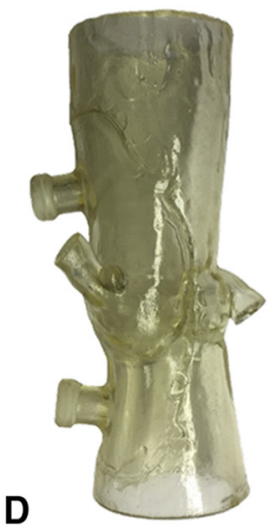

FIGURE 1. Steps followed to construct the 3-dimensional representative patient models. A, Computed tomography image segmentation. B, Aortic view of the aortic valve with leaflet calcification. C, Long-axis view of the digital model. D, Three-dimensional printed model. E, Aortic view of the 3-dimensional printed model. F, Left heart simulator flow loop where the valves are tested. $L C C$, left coronary cusp; NCC, noncoronary cusp; $R C C$, right coronary cusp.

the complex tissue properties of a diseased aortic root. The print material used for the noncalcified anatomic regions (TangoPlus) has a manufacturer-reported elastic modulus of $0.1 \mathrm{MPa}$ at $20 \%$ strain and $0.2 \mathrm{MPa}$ at $30 \%$ strain. The print material used for the calcified anatomic region (VeroWhitePlus) has a manufacturer-reported elastic modulus of 2000 to $3000 \mathrm{MPa}$.

Model 1 native annulus area and perimeter at mid-systole were measured to be $366 \mathrm{~mm}^{2}$ and $69.1 \mathrm{~mm}$, respectively. Model 2 valve native annulus area and perimeter at mid-systole were measured to be $616 \mathrm{~mm}^{2}$ and $90.5 \mathrm{~mm}$, respectively. In vitro representative patient modeling was done on the basis of the work of Maragiannis and colleagues ${ }^{15}$ and in-vitro experiments in our lab were performed using these models in Hatoum and colleagues. $^{16}$

\section{Valve Selection and Deployment}

To evaluate post-TAVR hemodynamics and sinus washout using self-expanding and balloon-expandable TAVs, measurements described next were conducted with a 26-mm Medtronic CoreValve TAV and a 23-mm Edwards SAPIEN TAV implanted in Model 1 root. Likewise, for Model 2 root, a 31-mm Medtronic CoreValve and a 29-mm Edwards SAPIEN S3 were deployed. The selection of the appropriate TAV was performed on the basis of anatomic factors and in conformity with the valve decision made by the structural heart team in the Wexner Medical Center based on the recommendations of Kasel and colleagues. ${ }^{17}$ The aorta was straight in our model without the aortic arch curvature, which is why the TAVs were implanted coaxially so there is no tilting. The deployment of the valves was done as recommended by Edwards ${ }^{18}$ and Medtronic ${ }^{19}$ to ensure optimal performance.

\section{Hemodynamic Assessment}

Hemodynamic parameters were evaluated under pulsatile flow conditions created by a left heart simulator (Figure 1, F) yielding physiologic flow and pressure curves as previously described. ${ }^{3,20,21}$ The working fluid in this study was a mixture of water-glycerin $(99 \%$ pure glycerine) producing a density of $1080 \mathrm{~kg} / \mathrm{m}^{3}$ and a kinematic viscosity of $3.5 \mathrm{cSt}$ similar to blood properties. Sixty consecutive cardiac cycles of aortic pressure, ventricular pressure, and flow rate data were recorded at a sampling rate of $100 \mathrm{~Hz}$. The mean transvalvular pressure gradient (PG) is defined as the average of positive pressure difference between the ventricular and aortic pressure curves during forward flow.

\section{Particle Image Velocimetry}

For particle image velocimetry (PIV), the flow was seeded with fluorescent PMMA-Rhodamine B particles with diameters ranging from 1 to $20 \mu \mathrm{m}$. For all cases, the velocity field within the sinus region including the region adjacent to the TAV leaflets were measured using high spatial and temporal resolution PIV. Briefly, this involved illuminating the sinus region using a laser sheet created by pulsed Nd:YLF single cavity diode pumped solid state laser coupled with external spherical and cylindrical lenses while acquiring high-speed images of the fluorescent particles within the sinus region. Raw PIV images were acquired with a resulting spatial and temporal resolutions of $0.02964 \mathrm{~mm} / \mathrm{pixel}$ and $4000 \mathrm{~Hz}$, respectively. Refraction was corrected using a calibration in DaVis PIV software (DaVis 7.2, LaVision Göttingen, Germany). Velocity vectors were calculated using adaptive cross-correlation algorithms. Further details of PIV measurements can be found in Hatoum and colleagues. $^{21,22}$ 


\section{Sinus Vorticity and Shear Stress Dynamics}

Vorticity quantifies local spinning of blood, and vorticity field illustrates how locally blood is rotating as it moves with a velocity and in what direction it is rotating (clockwise or counterclockwise) in the sinus. Vorticity dynamics are important because they give an idea about how the small scale particles move.

By using the velocity measurements from PIV, vorticity dynamics were also evaluated for the sinus region. Regions of high vorticity along the axis perpendicular to the plane indicate both shear and rotation of the fluid particles. Vorticity within the measurement region was computed using the following equation:

$$
\omega=-\left(\frac{d V_{x}}{d y}-\frac{d V_{y}}{d x}\right)
$$

where $\omega$ is the vorticity with units of $\mathrm{s}^{-1} ; V_{x}$ and $V_{y}$ are the $x$ and $y$ components of the velocity vector with units of $\mathrm{m} / \mathrm{s}$. The $x$ and $y$ directions are axial and lateral respectively with the $\mathrm{z}$ direction being out of measurement plane.

Viscous shear stress field was evaluated consistently with Moore and Dasi $^{5}$ and Hatoum and colleagues. ${ }^{3,21}$

$$
\tau=\mu\left(\frac{d V_{x}}{d y}+\frac{d V_{y}}{d x}\right)
$$

where $\tau$ is the shear stress in Pascal (Pa) and $\mu$ is the dynamic viscosity in N.s $/ \mathrm{m}^{2}$.

\section{Sinus Washout}

Velocity measurements from PIV were also used to evaluate sinus washout. Sinus washout is defined as the characteristic curve representing the percent of fluid particles, initially seeded in the sinus region at the beginning of the cardiac cycle, and still remaining in the sinus as a function of time plotted over the cardiac cycle. Ideally, good washout is associated with a high percentage of particles exiting over a minimum number of cardiac cycles. To quantify sinus washout curves, first particle tracking was performed similar to other studies. ${ }^{14,23,24}$ Briefly, particles were seeded as a uniform grid of $0.001 \mathrm{mx} 0.001 \mathrm{~m}$ cell size over the sinus region at the beginning of the cardiac cycle. Each particle's trajectory was computed by integrating its velocity with respect to time based on

$$
\frac{d \vec{x}}{d t}(t)=\vec{u}((\vec{x}), t)
$$

with

$$
\vec{x}(\mathrm{t}=0)=\vec{x}_{0}
$$

The integration time step was 0.00025 seconds, and at the end of every time step, the particle's velocity vector was calculated on the basis of the particle's updated location through interpolating the PIV velocity data.

After every cardiac cycle, only the particles that remained in the sinus were re-seeded based on their last positions, and their trajectory over the subsequent cardiac cycle was calculated. This process continued until all particles exited or until 10 cardiac cycles elapsed.

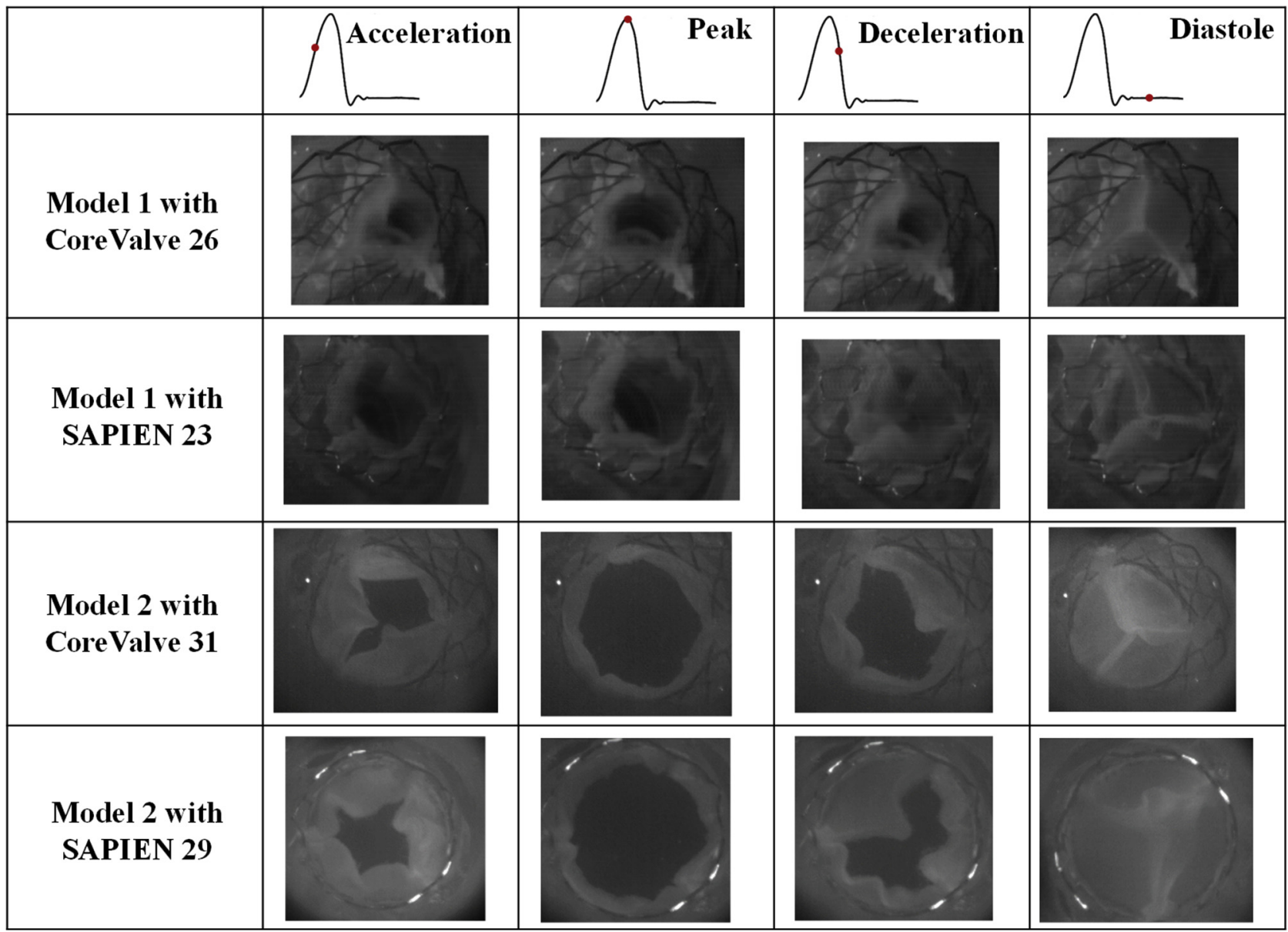

FIGURE 2. En face short axis imaging views of the valves at different phases in the cardiac cycle. 
Once all the particles exited the sinus, a histogram of the time spent by the particles was generated and then converted to a cumulative distribution function representing the particles' survival probability as a function of time. This procedure was repeated over 10 cycles for every valve combination. The resulting curves represent the sinus washout characteristic for all cases.

\section{Statistics}

All data are presented as mean \pm standard deviation. Student $t$ test was used to compare the means. Analyses were performed over 60 replicates. Probability density functions of the shear stress distribution were calculated and plotted. Sinus washout calculations were performed over 10 different cardiac cycles.

\section{RESULTS}

The sinus area that our study encompasses consists of the native sinus that bounds the neo-sinus as defined by Midha and colleagues. ${ }^{4}$ Leaflet thrombosis and flow stasis are evaluated on the basis of 3 different criteria that complement each other: (1) flow velocity fields, (2) shear stress probability distribution adjacent the TAV leaflets, and (3) washout in the whole sinus space.

\section{Flow Velocity Fields}

The en face views of the TAVs are shown in Figure 2 at key time points throughout the cardiac cycle showing how the TAV leaflets open and close within the representative patient anatomy. Figure 3 shows the velocity vectors and vorticity contours within the 2 different representative patient roots post-TAVR with CoreValve 26 and 31 and SAPIEN 23 and 29 at selected time points throughout the cardiac cycle. As shown in Figure 3, vorticity patterns and magnitude change in the sinus between every case with TAV type and patient characteristics.

The maximum velocity at peak systole in Model 1 is $0.13 \pm 0.01 \mathrm{~m} / \mathrm{s}$ when a CoreValve is implanted and $0.30 \pm 0.02 \mathrm{~m} / \mathrm{s}$ when a SAPIEN is implanted $(P<.01)$. In the same manner, the vorticity magnitude is $75 \pm 1.1 \mathrm{~s}^{-1}$ and $109 \pm 2.3 \mathrm{~s}^{-1}$ with the CoreValve and SAPIEN, respectively $(P<.01)$. The velocity reaches $0.02 \pm 0.005 \mathrm{~m} / \mathrm{s}$ and $0.07 \pm 0.003 \mathrm{~m} / \mathrm{s}$ during mid-diastole with CoreValve and SAPIEN, respectively. The velocity at the intersection between the native sinus and the neo-sinus equal to $0.02 \pm 0.012 \mathrm{~m} / \mathrm{s}$ with a CoreValve and $0.08 \pm 0.015 \mathrm{~m} / \mathrm{s}$ with a SAPIEN $(P<.01)$. After TAVR, blood flow tends to leave the sinus instead of maintaining the aortic sinus vortex throughout the cardiac cycle.

The maximum velocity at peak systole in Model 2 reaches $0.10 \pm 0.02 \mathrm{~m} / \mathrm{s}$ when a CoreValve is implanted and $0.34 \pm 0.041 \mathrm{~m} / \mathrm{s}$ when a SAPIEN is implanted $(P<.01)$. In the same manner, the vorticity magnitude is $77 \pm 3.2 \mathrm{~s}^{-1}$ and $250 \pm 4.1 \mathrm{~s}^{-1}$ with CoreValve and with SAPIEN, respectively $(P<.01)$. The velocity reaches $0.024 \pm 0.003 \mathrm{~m} / \mathrm{s} 0.073 \pm 0.005 \mathrm{~m} / \mathrm{s}$ with CoreValve and SAPIEN during mid-diastole, respectively. The velocity at the intersection between the native sinus and the neo-sinus equal to $0.015 \pm 0.004 \mathrm{~m} / \mathrm{s}$ with a CoreValve and $0.04 \pm 0.01 \mathrm{~m} / \mathrm{s}$ with a SAPIEN $(P=.42)$. The vortex propagation in the sinus is shown post-TAVR. However, it is less prevalent in the CoreValve case, as shown in Figure 3 and less vigorous in the SAPIEN case.

The sinus velocities in the 2 different representative patient models with the CoreValves were shown to be

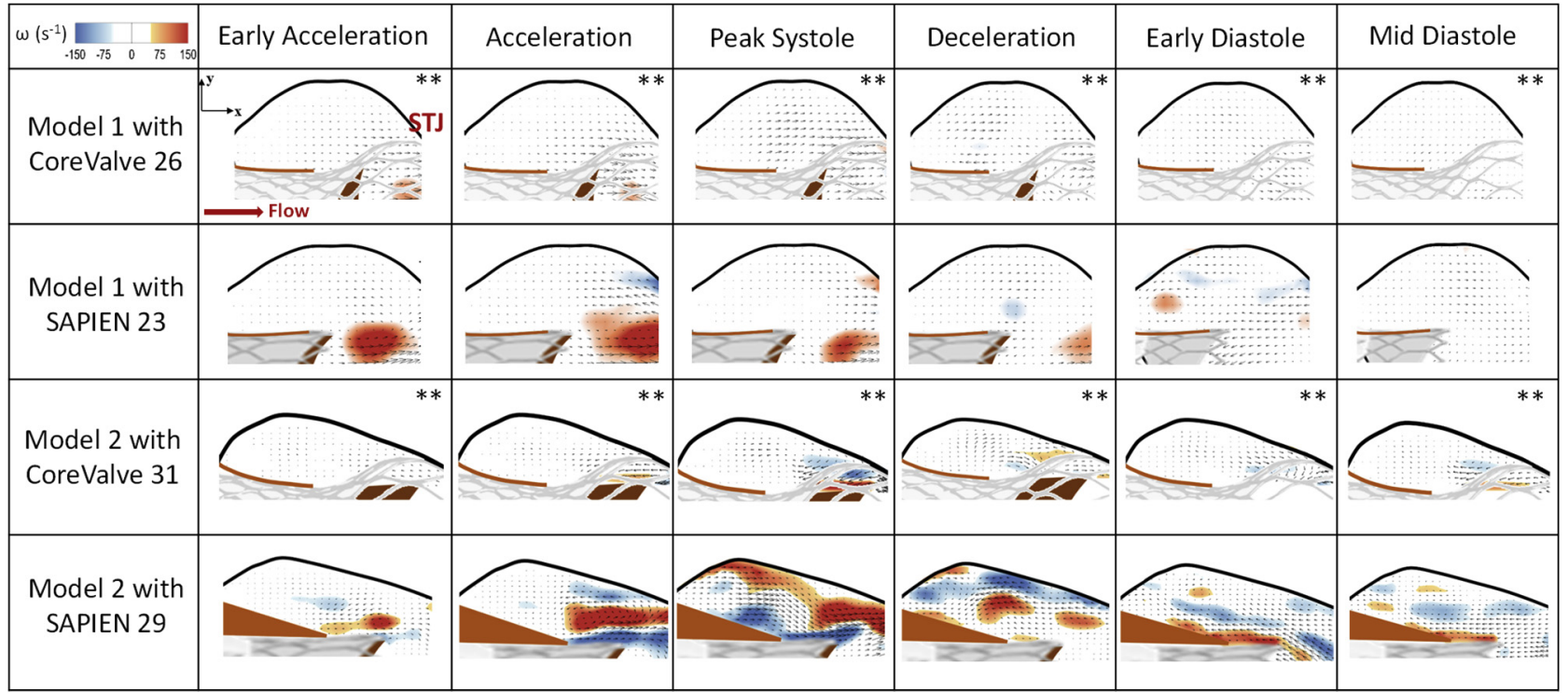

FIGURE 3. Velocity vectors and vorticity contours within the representative patient models 1 and 2 post-TAVR with CoreValve 26 and 31 (Medtronic Inc, Irvine, Calif) and SAPIEN 23 and 29 (Edwards Lifesciences, Irvine Calif) at selected time points throughout the cardiac cycle. STJ, Sinotubular junction. **The vector length is 3 times higher. 

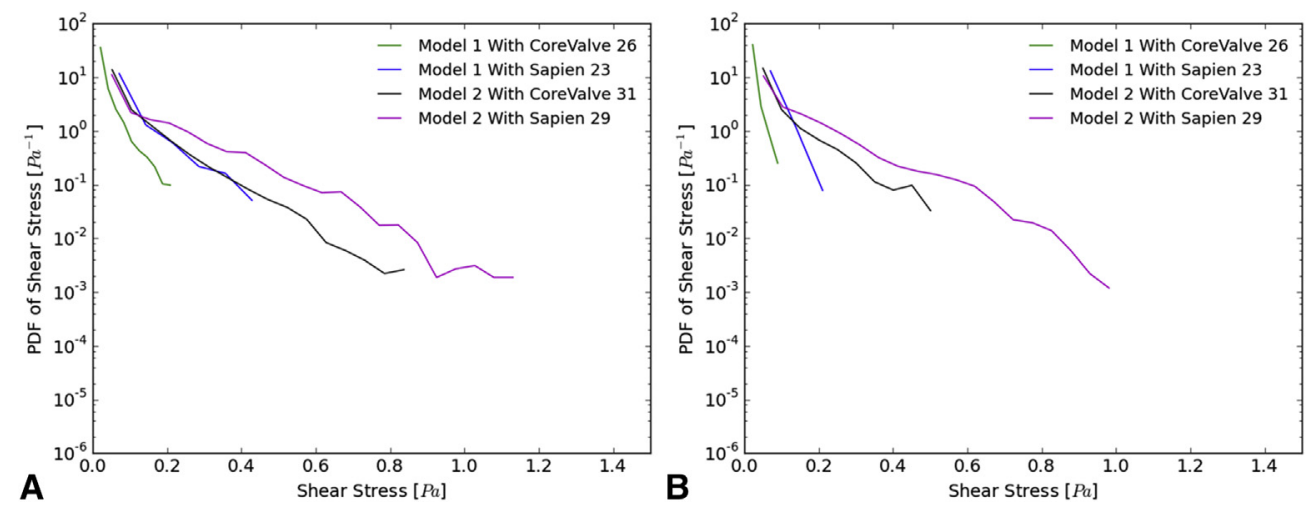

FIGURE 4. Probability density function in log scale of varying shear stress distribution values along a subregion near the valve leaflets during (A) systole and (B) diastole for the 2 representative patient models. $P D F$, Probability density function.

insignificant $(P=.08)$, as well as with the SAPIENs $(P=.19)$. The vorticities with the CoreValves were shown to be insignificant $(P=.28)$, but they were significant with the SAPIEN $(P<.01)$, highlighting the patient root interaction with the TAVs.

\section{Shear Stress Distribution}

Figure $4, A$ and $B$, show the probability density function of flow shear stress magnitude in the sub-region adjacent to the leaflets during systole and diastole, respectively. The region studied post-TAVR extends from the native leaflets to the tip of the bioprosthetic leaflet in the neo-sinus. It is also clear that higher shear stress magnitudes are obtained during systole compared with diastole.

For Model 1 during systole, after SAPIEN and CoreValve implantation, the likelihood of having high shear stress near the leaflet decreases drastically $(<0.4 \mathrm{~Pa})$; however, the SAPIEN yields higher values (up to $0.4 \mathrm{~Pa}$ ) compared with the CoreValve (up to $0.2 \mathrm{~Pa}$ ). The SAPIEN implanted in Model 2 is associated with higher shear stresses (up to 1.2 Pa) during systole.

During diastole, the maximum shear stress levels reach approximately $0.25 \mathrm{~Pa}$ for the SAPIEN in Model 1 and $0.15 \mathrm{~Pa}$ for the CoreValve. For Model 2, the CoreValve yields lower probabilities of high shear stresses near the leaflets $(\sim 0.5 \mathrm{~Pa})$, and the SAPIEN yields approximately $0.95 \mathrm{~Pa}$. Whether in systole or diastole, both TAVs perform better in Model 2 than Model 1 one, highlighting the patient root interaction with the TAVs.

\section{Sinus Washout}

Figure 5 shows the survival probability curve of particles remaining in both representative patient sinuses with different valve combinations.

When a CoreValve is implanted in Model 1, 69\% of the particles exit the sinus (31\% remain) after the first cardiac cycle, followed by an extra $21 \%$ at the end of the second cardiac cycle. The decrease after that becomes gradual to reach $0 \%$ of particle remaining in the sinus after approximately 3.5 cycles. When a SAPIEN is implanted, $96.2 \%$ of the particles exit the sinus region (3.8\% remain) after the first cycle and the particles are totally washed out at 1.25 cycles.

When a CoreValve is implanted in Model 2, 37.82\% of the particles exit $(62.18 \%$ remain) after the first cardiac cycle, followed by an additional $18.48 \%$ by the end of the second cycle. At the end of the 10 cycles, $18.17 \%$ of the particles remain, yielding a washout of $81.52 \%$. When a SAPIEN is implanted, $75 \%$ of the particles exit (25\% remain) the sinus after the first cardiac cycle, followed by an additional 3\% by the end of the second cardiac cycle. After 7.5 cycles, all the particles are washed out.

By comparing the TAVs in every representative patient root model, the washout seems to be better in Model 1 compared with Model 2 regardless of the TAV, highlighting

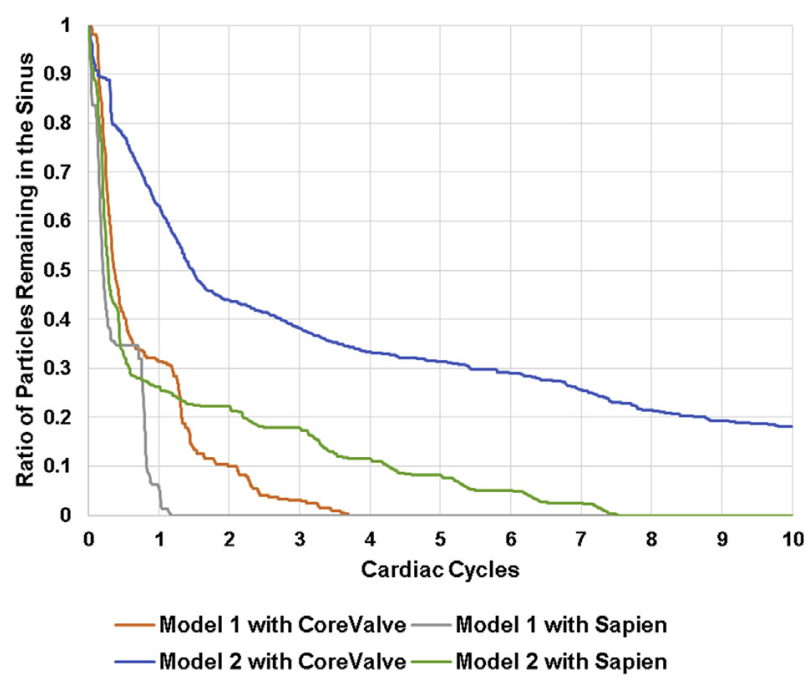

FIGURE 5. Survival probability curve of particles remaining in model 1 sinus and model 2 sinus post-TAVR with different valve combinations. 
the potential dominance of the representative patient root interaction with the TAVs.

\section{DISCUSSION}

The results of this work for the first time capture the complex hemodynamics within the aortic sinus post-TAVR in representative patient models to provide new perspectives of various mechanisms at play in the contexts of TAVR-related leaflet thrombosis. The basic physics of sinus vortex continues to be the same as described by Peacock. ${ }^{25}$ Specifically during forward flow period, the forward jet is bound by a free shear layer after exiting from the orifice defined by the tip of the fully open TAV leaflets. When the shear layer is intercepted by the sinus ridge (ie, the point connecting the sinus to the STJ), a portion of the flow curls back toward the ventricle thereby driving a spinning vortex within each sinus cavity. ${ }^{25}$ However, the precise nature of the vortex (single or multiple), spatio-temporal evolution over the cardiac cycle, and the overall strength are greatly dependent on (1) the geometry of the sinus cavity, which is defined by the aortic root lumen and the calcified native leaflets that can influence valve seating in relation to the root. Heavy calcification and calcification pattern in patients are influencing factors in addition to anatomic variations in the leaflets themselves; (2) the presence of the TAV prosthesis elements such as stent frames, (3) the relative position of the TAV leaflet tip in relation to the sinus ridge, and (4) the strength of the forward flow. Although previous studies attempted to characterize the aortic sinus vortex experimentally ${ }^{26}$ and numerically, ${ }^{27,28}$ there are no studies that examined the influence of sinus flow and vortex propagation with respect to representative patient geometry and TAV interaction. Many publications have highlighted the complexity of the flow inside the sinus stemming from the existence of small time scale vortices in addition to a main vortex. ${ }^{2,29}$ A numeric simulation by Fukui and Morinishi ${ }^{30}$ has emphasized not only the presence of many vortices but also the effect of sinus morphology such as extension and bulge depth on the formation and propagation of vortices and flow inside the sinus. In what follows, we discuss the detailed sinus vortex measurements in this study and examine how sensitive the measures of shear stress as well as sinus washout are with respect to patient root morphology and TAV type.

\section{Flow Velocity Fields}

It was previously shown that TAV deployment alters sinus flow patterns by significantly decreasing sinus velocity and vorticity for both valve types whether the SAPIEN or CoreValve. ${ }^{3}$ In addition, it modifies the vortex propagation mechanism in the different valve cases. ${ }^{3,21}$

When a CoreValve is implanted, its particular stent mesh plays a role in changing the mechanics of the vortex formation and propagation. A study by Adhikari and $\mathrm{Lim}^{31}$ showed that a porous screen permits the self-induced velocity of the primary vortex ring to pass through it to form a jet-like flow. This transmitted jet carries with it some of the original vorticity, leaving behind a weaker primary vortex ring to interact with the screen. As a consequence, a weaker secondary vortex ring is generated, resulting in a weaker vortex. When the SAPIEN is implanted, the overall flow pattern resembles that seen in the CoreValve case where the fluid flow tends to leave both the native sinus and the neo-sinus slowly instead of rotating. So at the central part of the sinus (Figure 3), the fluid motion is toward exiting the whole sinus region, and from the middle to the leaflet level, the fluid motion is more rotational. The SAPIEN represents a better scenario than CoreValve. Having the leaflet tip position more upstream than that of the CoreValve, the vortex that starts forming does not engender a whole fluid motion throughout the sinus. However, it does cause the rotation and the main motion in the region adjacent to the STJ while leading the flow in the middle of the sinus to exit the area.

The SAPIEN TAV showed higher (thus more favorable) velocities and vorticities compared with the CoreValve in both sinus cases. In addition, having a SAPIEN implanted improves significantly the rotation in this particular representative patient Model 2 compared with Model 1. These observations may be due to the shorter profile of the SAPIEN allowing for more space to be occupied by the fluid thus easing the flow. Also, the closer leaflet tip position toward the annulus than the STJ allows more space for vortices to form and flow to move into the sinus easily with minimum obstruction. This highlights the importance of the variability of TAV representative patient anatomy interaction, which involves not only the interaction with the root but also with the calcified leaflets. The interaction with the leaflets further involves not just calcification distribution but also the morphology of the leaflets (eg, bicuspid vs tricuspid) that can influence valve seating. The CoreValve did not show significant variations between the 2 models.

\section{Shear Stress Distribution}

Thrombosis is most likely to occur in low-flow or stasis regions with reduced and oscillatory shear stresses. ${ }^{32,33}$ In healthy blood vessels, shear stress values range from 1.5 to $2 \mathrm{~Pa}$. Shear stress usually varies with the local conditions and the flow rate. Low values of shear stress or tremendous variation in values (eg, oscillatory) changes the behavior of some cells (eg, platelets) and can lead to thrombus formation. ${ }^{34}$ It is the shear-dependent mass transport that is responsible for atheroma growth and thus higher risk of thrombosis. Furthermore, the endothelium has been shown to become atherogenic when exposed to low wall shear stress. ${ }^{34,35}$ Several studies have reported 
and classified shear stress values in grafts as "high" and "low," 36 and suggested low values of shear stress to be $0.25 \mathrm{~Pa}$ and $0.31 \mathrm{~Pa}$, and the high values were $1.54 \mathrm{~Pa}$ and $1.71 \mathrm{~Pa}$. Another study of vascular shear stress by Cunningham and Gotlieb ${ }^{7}$ showed that vascular shear stress of large conduit arteries typically varies between 5 and 20 dynes $/ \mathrm{cm}^{2}$ ( 0.5 to $2.0 \mathrm{~Pa}$ ). Another study by Casa and colleagues $^{8}$ reported a normal value of $1000 \mathrm{~s}^{-1}$ for shear rate that corresponds to $3.5 \mathrm{~Pa}$ in arteries and a value of $500 \mathrm{~s}^{-1}$ corresponding to $1.75 \mathrm{~Pa}$ in coronary arteries. A study by Bark and colleagues ${ }^{9}$ has reported physiologic arterial shear rates below $400 \mathrm{~s}^{-1}$ equivalent to $1.4 \mathrm{~Pa}$.

The fact that the probability of developing high shear stresses tremendously varies nearby the leaflets once a TAV is implanted in the 2 different representative patient aortic valve models underscores the complexities associated with the selection of the appropriate $\mathrm{TAV}^{3,21,37}$ It is clear that shear stress distribution near the leaflet whether in systole or diastole in a function of TAV type and representative patient anatomy. The SAPIEN performs better in terms of shear stress than the CoreValve in both models. Both TAVs perform better in Model 2 than Model 1, highlighting the importance of representative patient interaction with the TAVs. It may also be due to how the sinus connects with the STJ and at what level. A lower STJ-sinus connection point allows for more vortex entrapment thus constant movement inside the sinus (Model 1) unlike a higher one that allows more exchange of flow (Model 2).

In diastole, the greatly decreased shear stress level in Model 1 sinus near the leaflet after TAVR connects well with the slowed down flow previously observed. Such a large reduction in shear stress could lead to thrombus growth and associated leaflet mobility problems. $3,9,38$

\section{Sinus Washout}

Thrombosis is most likely to occur in low-flow or stasis regions characterized by longer particle/cell residence time. $^{39,40}$ The implantation of a TAV aims at improving the overall sinus washout with the least cardiac cycles possible. ${ }^{21}$ The SAPIEN improved washout drastically after the first and second cardiac cycles compared to the CoreValve. The sinus region, when a SAPIEN is implanted, shows better distributions of vortical structures leading to more efficient fluid motion than the CoreValve.

The CoreValve and the SAPIEN seem to improve the washout better in Model 1 sinus compared with Model 2 emphasizing the complexity of the anatomy and TAV interaction, especially that the shear stress distribution near the leaflet as previously described gets tremendously reduced in Model 1 sinus compared with that of Model 2. Also note that the curves for Model 1 represent plateaus followed by steep drops indicating that washout appears to occur in intense events of blood exiting the sinus compared with that in Model 2, which shows a gradual washout without intense events. Although velocities did not seem to be significantly different among the models with the same valve, the rotation in the SAPIEN was significantly higher in Model 2 sinus compared with Model 1. This higher rotation may explain the poorer washout characteristic while still maintaining higher shear stress events. These findings highlight the importance of how the TAV behaves within its surroundings and how the impact of that complicates the hemodynamics in that shear stress and washout appear to be independent measures with both necessary to examine stasis.

\section{Comparison Between Idealized and Anatomic Sinus Geometries}

To the best of our knowledge, this is the first study of sinus flow patterns complexity in an anatomic representative patient model post-TAVR. Many previous studies have shown that the implantation of a TAV does not affect the formation of flow patterns inside the idealized sinus, but the patterns were weaker. ${ }^{6}$ These sinus flow patterns were comparable in the native aortic valves, bioprosthetic valves, polymeric valves, and Lotus valve. ${ }^{6,41-43}$ The common line between these studies was adopting an idealized axisymmetric design of the aortic sinus (ie, absence of 3 sinuses). In this study, as highlighted in the previous discussion points, the different anatomic patient morphologies contributed to profoundly different dynamics starting from different durations of formation and propagation of the vortex, different vortex entrapment patterns, and different vortex dissipation and fission to smaller vortices. These differences engendered different washout, different velocity fields, and therefore shear stress distributions nearby the leaflets. The sinus vortex dynamics highly influence stasis regions, thus highlighting the importance of patient characteristics and the TAV interaction with them.

As a final discussion point, the complex flow patterns in the sinus that are inherently valve- and patient-specific lead to mechanical forces at scales that can cause blood cell damage and stasis, thus probably thrombosis, which can trigger the pathogenesis of various valvular heart diseases. From a comprehensive perspective, the linkages between valve hemodynamics and disease pathways as well as clinical complications require the use of integrated multimodality approaches that incorporate information across a range of scales and bridge the gap between mechanics and biological cascades. Although molecular pathways have a vital and crucial role when it comes to thrombus formation, our study only uses 1 multi-modality approach that is looking into hemodynamics through flow fields.

\section{Study Limitations}

This study considered only 2 representative patient aortic root models that encompass a combination of parameters 
that we cannot isolate (variability in terms of the size and shape of the annulus, size and height of the sinuses, the size of the STJ, the height of the coronaries and dominance, the size of the aorta, and the size and shape of the LVOT). In addition, only the Edwards SAPIEN and Medtronic Evolut TAVs were tested in a way that their commissures align with those of the native valve (for which there is no way to ensure that this is the case in vivo during the procedure). This study also only presented the dynamics in the noncoronary sinus hemodynamics (as a conservative representation from the standpoint of stasis). However, the purpose of the study is to illustrate how sensitive sinus hemodynamics are after TAVR with the complex TAV-patient anatomy interaction (even for the same valve). Because this study does not represent any population or group study, generalized conclusions about valve choice in relation to the anatomic features and the ensuing shear stress washout characteristics should be avoided. Further studies with coronary flow are needed to provide a more complete picture.

\section{CONCLUSIONS}

This study presents a detailed assessment of sinus hemodynamics in 2 different representative patient aortic roots after TAVR. Novel methodology was developed to simulate in vitro conditions as close as possible to the physiologic ones using representative aortic valve roots segmented and 3-dimensionally printed from a patient's computed tomography angiogram. It is shown that overall sinus flows are greatly altered post-TAVR with CoreValve versus SAPIEN in the 2 models. Specifically, the SAPIEN always yielded more favorable shear stress and washout characteristics. However, the interaction of either TAVs with the 2 different roots can greatly affect both shear stress and washout characteristics with the fundamental mechanism always being about the alteration of the sinus vortex formation and propagation. This study highlights the complexity of patient factors through looking at 2 different anatomic roots, and their TAV interaction that may constitute a rich variety of factors to consider when assessing potential risk of leaflet thrombosis in patients.

\section{Conflict of Interest Statement}

J.C. reports grants from Medtronic, Boston Scientific and St Jude in addition to being part of the advisory board of Medtronic. L.P.D. reports a patent application filed on novel polymeric valves. All other authors have nothing to disclose with regard to commercial support.

\section{References}

1. Toninato R, Salmon J, Susin FM, Ducci A, Burriesci G. Physiological vortices in the sinuses of Valsalva: An in vitro approach for bio-prosthetic valves. J Biomech. 2016;49:2635-43.

2. Moore B, Dasi LP. Spatiotemporal complexity of the aortic sinus vortex. Exp Fluids. 2014;55:1770.
3. Hatoum H, Moore BL, Maureira P, Dollery J, Crestanello JA, Dasi LP. Aortic sinus flow stasis likely in valve-in-valve transcatheter aortic valve implantation. J Thorac Cardiovasc Surg. 2017;154:32-43.e1.

4. Midha PA, Raghav V, Sharma R, Condado JF, Okafor IU, Rami T, et al. The fluid mechanics of transcatheter heart valve leaflet thrombosis in the neo-sinus. Circulation. 2017;136:1598-609.

5. Moore BL, Dasi LP. Coronary flow impacts aortic leaflet mechanics and aortic sinus hemodynamics. Ann Biomed Eng. 2015;43:2231-41.

6. Saikrishnan N, Gupta S, Yoganathan AP. Hemodynamics of the Boston Scientific Lotus ${ }^{\mathrm{TM}}$ valve: an in vitro study. Cardiovas Eng Technol. 2013;4:427-39.

7. Cunningham KS, Gotlieb AI. The role of shear stress in the pathogenesis of atherosclerosis. Lab Invest. 2005;85:9.

8. Casa LD, Deaton DH, Ku DN. Role of high shear rate in thrombosis. J Vasc Surg. 2015;61:1068-80.

9. Bark DL, Para AN, Ku DN. Correlation of thrombosis growth rate to pathological wall shear rate during platelet accumulation. Biotechnol Bioeng. 2012;109: 2642-50.

10. Makkar RR, Fontana G, Jilaihawi H, Chakravarty T, Kofoed KF, De Backer O, et al. Possible subclinical leaflet thrombosis in bioprosthetic aortic valves. $N$ Engl J Med. 2015;373:2015-24.

11. Chakravarty T, Søndergaard L, Friedman J, De Backer O, Berman D, Kofoed KF, et al. Subclinical leaflet thrombosis in surgical and transcatheter bioprosthetic aortic valves: an observational study. Lancet. 2017;389:2383-92.

12. Azadani AN, Reardon M, Simonato M, Aldea G, Nickenig G, Kornowski R, Dvir D. Effect of transcatheter aortic valve size and position on valve-in-valve hemodynamics: an in vitro study. J Thorac Cardiovasc Surg. 2017;153: 1303-15.e1.

13. Bapat VN. Effect of transcatheter aortic valve size and position on valve-invalve hemodynamics: an in vitro study. J Thorac Cardiovasc Surg. 2017;154: 58-9.

14. Midha PA, Raghav V, Okafor I, Yoganathan AP. The effect of valve-in-valve implantation height on sinus flow. Ann Biomed Eng. 2016;1-8.

15. Maragiannis D, Jackson MS, Igo SR, Schutt RC, Connell P, Grande-Allen J, et al. Replicating patient-specific severe aortic valve stenosis with functional 3D modeling clinical perspective. Circ Cardiovasc Imaging. 2015;8:e003626.

16. Hatoum H, Yousefi A, Lilly S, Maureira P, Crestanello J, Dasi LP. An in vitro evaluation of turbulence after transcatheter aortic valve implantation. J Thorac Cardiovasc Surg. 2018;156:1837-48.

17. Kasel AM, Cassese S, Bleiziffer S, Amaki M, Hahn RT, Kastrati A, Sengupta PP. Standardized imaging for aortic annular sizing. JACC Cardiovasc Imaging. 2013; 6:249-62.

18. Edwards Lifesciences Corporation. TAVR with the Sapien 3 valve. Available at: https://www.edwards.com/devices/heart-valves/transcatheter-Sapien-3. Accessed June 26, 2018.

19. Medtronic. CoreValve in situ. Available at: http://media.corporate-ir.net/media files/IROL/25/251324/Images/CoreValve\%20in\%20situ.jpg. Accessed June 26, 2018.

20. Forleo M, Dasi LP. Effect of hypertension on the closing dynamics and lagrangian blood damage index measure of the B-Datum Regurgitant Jet in a bileaflet mechanical heart valve. Ann Biomed Eng. 2014;42:110-22.

21. Hatoum H, Dollery J, Lilly SM, Crestanello JA, Dasi LP. Implantation depth and rotational orientation effect on valve-in-valve hemodynamics and sinus flow. Ann Thorac Surg. 2018;106:70-8.

22. Hatoum H, Dollery J, Lilly SM, Crestanello JA, Dasi LP. Effect of severe bioprosthetic valve tissue ingrowth and inflow calcification on valve-in-valve performance. J Biomech. 2018;74:171-9.

23. Vahidkhah K, Azadani AN. Supra-annular valve-in-valve implantation reduces blood stasis on the transcatheter aortic valve leaflets. J Biomech. 2017;58:114-22.

24. Kumar G, Raghav V, Lerakis S, Yoganathan AP. High transcatheter valve replacement may reduce washout in the aortic sinuses: an in-vitro study. J Heart Valve Dis. 2015;24:22-9.

25. Peacock JA. An in vitro study of the onset of turbulence in the sinus of Valsalva. Circ Res. 1990;67:448-60.

26. Bellhouse BJ, Talbot L. The fluid mechanics of the aortic valve. J Fluid Mech. 1969;35:721-35.

27. Gillani NV, Swanson W. Time-dependent laminar incompressible flow through a spherical cavity. J Fluid Mech. 1976;78:99-127.

28. Peskin C, Wolfe A. The aortic sinus vortex. Fed Proc. 1978;2784-92.

29. Ducci A, Tzamtzis S, Mullen MJ, Burriesci G. Hemodynamics in the Valsalva sinuses after transcatheter aortic valve implantation (TAVI). J Heart Valve Dis. 2013;22:688-96. 
30. Fukui T, Morinishi K. Influence of vortices in the sinus of Valsalva on local wall shear stress distribution. Int J Life Sci Med Res. 2013;3:94.

31. Adhikari D, Lim T. The impact of a vortex ring on a porous screen. Fluid Dyn Res. 2009;41:051404.

32. Dasi LP, Hatoum H, Kheradvar A, Zareian R, Alavi SH, Sun W, et al. On the mechanics of transcatheter aortic valve replacement. Ann Biomed Eng. 2017;45: 310-31.

33. Chandra S, Rajamannan NM, Sucosky P. Computational assessment of bicuspid aortic valve wall-shear stress: implications for calcific aortic valve disease. Biomech Model Mechanobiol. 2012;11:1085-96.

34. Traub O, Berk BC. Laminar shear stress: mechanisms by which endothelial cells transduce an atheroprotective force. Arterioscler Thromb Vasc Biol. 1998;18: 677-85.

35. Berk BC, ABE JI, Min W, Surapisitchat J, Yan C. Endothelial atheroprotective and anti-inflammatory mechanisms. Ann NY Acad Sci. 2001;947:93-111.

36. Wu MH, Kouchi Y, Onuki Y, Shi Q, Yoshida H, Kaplan S, et al. Effect of differential shear stress on platelet aggregation, surface thrombosis, and endothelialization of bilateral carotid-femoral grafts in the dog. J Vasc Surg. 1995:22:382-92.

37. Yoon S-H, Bleiziffer S, De Backer O, Delgado V, Arai T, Ziegelmueller J, et al. Outcomes in transcatheter aortic valve replacement for bicuspid versus tricuspid aortic valve stenosis. J Am Coll Cardiol. 2017;69:2579-89.
38. Hatoum H, Crestanello JA, Dasi LP. Possible subclinical leaflet thrombosis in bioprosthetic aortic valves. N Engl J Med. 2016;374:1591.

39. Rayz VL, Boussel L, Ge L, Leach JR, Martin AJ, Lawton MT, et al. Flow residence time and regions of intraluminal thrombus deposition in intracranial aneurysms. Ann Biomed Eng. 2010;38:3058-69.

40. Gorbet MB, Sefton MV. Biomaterial-associated thrombosis: roles of coagulation factors, complement, platelets and leukocytes. Biomaterials. 2004; 25:5681-703.

41. Saikrishnan N, Yap C-H, Milligan NC, Vasilyev NV, Yoganathan AP. In vitro characterization of bicuspid aortic valve hemodynamics using particle image velocimetry. Ann Biomed Eng. 2012;40:1760-75.

42. Yoganathan A, Woo Y, Sung H, Williams F, Franch R, Jones M. In vitro hemodynamic characteristics of tissue bioprostheses in the aortic position. J Thorac Cardiovasc Surg. 1986;92:198-209.

43. Leo HL, Dasi LP, Carberry J, Simon HA, Yoganathan AP. Fluid dynamic assessment of three polymeric heart valves using particle image velocimetry. Ann Biomed Eng. 2006;34:936-52.

Key Words: leaflet thrombosis, patient root, sinus flow, stasis, TAVR, transcatheter aortic valve 